\title{
Colon Cancer Detection by 'Rendezvous Colonoscopy': Successful Removal of Stuck Colon Capsule by Conventional Colonoscopy
}

\author{
István Rácz Márta Jánoki Hussam Saleh \\ Department of Gastroenterology, Petz Aladár County and Teaching Hospital, \\ Győr, Hungary
}

\section{Key Words}

Colon cancer detection · 'Rendezvous colonoscopy' · Stuck colon capsule · Conventional colonoscopy

\begin{abstract}
Although capsule retention is a known complication of small bowel capsule endoscopy, initial studies with colon capsule endoscopy (CCE) have not reported any capsule retention or sticking neither in the small bowel nor in the colon. We report a complication of CCE when the stuck colon capsule was passed through the malignant colon stricture and removed by the aid of a flexible colonoscope. During CCE in a 76-year-old iron deficiency anemia patient the real-time viewing system indicated the colon capsule to be stuck in a malignant ascending colon stricture for more than two hours. With the aim to avoid complete capsule retention, immediate colonoscopy was performed. The stuck capsule was caught by a polypectomy snare, passed through the tumor stricture and finally removed from the large bowel. The current case describes the usefulness of the real-time viewing system. Similar situations may occur during the forthcoming spread of CCE and the present case is an example of how to manage the potentially risky stuck colon capsule condition.
\end{abstract}

\section{Introduction}

In initial studies colon capsule endoscopy (CCE) has been shown to be safe and able to demonstrate colon polyps and cancers [1-3]. In these CCE studies there were no severe adverse events and the comparative colonoscopies were performed the same day as the CCE, after excretion of capsule or when recording of images had stopped. We report a 
special case of CCE where the stuck colon capsule was passed through the malignant colon stricture and removed by the aid of flexible colonoscopy.

\section{Case Report}

A 76-year-old woman with a history of percutaneous coronary stent implantation was admitted because of worsening dyspnea and cardiac chest pain. Medications included $75 \mathrm{mg}$ clopidogrel and $100 \mathrm{mg}$ aspirin daily. At admission $\mathrm{Hb}$ was $4.8 \mathrm{~g} / \mathrm{dl}$ (normal $12-16 \mathrm{~g} / \mathrm{dl}$ ), all other routine laboratory tests were within normal ranges. There was no hematochezia or melena, but fecal occult blood test was positive. The patient received 6 units of packed red cells transfusion. Esophagogastroduodenoscopy was negative for a source of bleeding. With the suspicion of large bowel bleeding origin, PillCam Colon capsule endoscopy (Given Imaging Ltd., Yoqneam, Israel) was chosen initially because of its noninvasive nature. The patient had neither previous intestinal surgery nor symptoms for suspected bowel obstruction and abdominal ultrasound was negative.

A 24-hour colon preparation with $6 \mathrm{l}$ of an oral preparation was performed of polyethylene glycol osmotic solution (Colopeg, Roche Laboratories, Gaillard, France) without any adverse event. The colon capsule reached the colon after 69 min small bowel transit time. During the CCE procedure real-time viewing was performed with Rapid Access Real Time Tablet PC (Given Imaging Inc.) every 15 min to determine the location of the capsule. By this method an actively bleeding obstructive tumor mass was detected in the ascending colon which blocked the capsule passage for $2.5 \mathrm{~h}(\underline{\mathrm{fig}} .1,2)$. At this point, with the aim to avoid total bowel obstruction with clinical emergency and also to complete the diagnosis, an immediate colonoscopy was performed with a standard colonoscope (CF-160, Olympus, Tokyo, Japan). Colonoscopy revealed a $10-\mathrm{cm}$-long ascending colon tumor leaving only a $10-12 \mathrm{~mm}$ lumen in diameter at the most oral tumor segment. Although by standard colonoscopy it was possible to intubate only the distal part of the tumorous stenosis, there was a chance to cannulate the most narrow proximal segment with a polypectomy snare. As the capsule was stuck in a partly transverse position, it was first mobilized to a longitudinal position, then caught by the polypectomy snare and passed through the tumorous stricture (fig. 3 ). After taking biopsies the capsule was removed from the colon (fig. 4). Histology showed grade II adenocarcinoma. The patient underwent right side hemicolectomy without complication and was discharged home on hospital day 14.

\section{Discussion}

Capsule endoscopy has rapidly expanded for the visualization of the small intestine and CCE appears to be a promising new modality for colonic evaluation. Although capsule retention is one of the known complications of small bowel capsule endoscopy [4-6], initial studies with CCE, despite the larger capsule size $(11 \times 31 \mathrm{~mm})$, have not reported any capsule retention or sticking neither in the small bowel nor in the colon. In our high cardiological risk case, after negative esophagogastroduodenoscopy CCE was chosen first for colon examination, however conventional colonoscopy was also available as a standby method. Assuming the experiences of published CCE studies, if conventional colonoscopy is indicated based upon the CCE results, the most appropriate is to perform it on the same day as the capsule study when the colon is already prepared. The real-time viewing system offers occasional control of capsule localization and progression, and moreover, as in our case, the immediate detection of suspected bleeding lesions.

Capsule retention is defined as the presence of the capsule endoscope in the digestive tract for a minimum of 2 weeks [6,7]. In our case the colon capsule was stuck in the malignant colon stricture for more than two hours, obviously not fulfilling the criteria of capsule retention. However, there is no data available yet about the usual clinical outcome of stuck colon capsule situations. Although colon capsules operate about $10 \mathrm{~h}$ and in our case we did not know whether the stuck capsule would pass spontaneously through the stricture, we decided to perform immediate colonoscopy to avoid complete bowel obstruction resulting in clinical emergency. Assuming all conditions we felt that for our 
high age cardiac patient it is less risky to perform flexible colonoscopy as soon as possible instead of monitoring the possible capsule retention. Polypectomy snare proved to be a suitable device for mobilizing, catching and removing the capsule. Considering that the colon capsule was stuck transversely in the significant stricture, we concluded that not the short examination time but rather the anatomical situation was the reason of incomplete capsule colonoscopy.

Several case presentations combining capsule endoscopy with colonoscopy have been published. Riccioni et al. [8] detected a bleeding colon cancer with small bowel capsule while Spada et al. [9] reported a case in whom CCE allowed a complete colonoscopy that was impossible initially with conventional endoscopy. Triantafyllou et al. [10] in a retrospective case series found that in only half of the patients with incomplete colonoscopy CCE offered a satisfactory colon examination. To our knowledge this is the first case presentation demonstrating the combination of CCE and immediate conventional colonoscopy to the diagnosis of bleeding colon cancer and the successful removal of a stuck capsule.

The current case describes the usefulness of the real-time viewing system and the methodology of 'rendezvous colonoscopy'. We believe that by the forthcoming spread of CCE similar clinical situations may occur and that endoscopists should be aware of them to manage potentially risky stuck colon capsule conditions.

Fig. 1. PillCam Colon capsule endoscopic view of the ascending colon tumor with significant lumen obstruction.

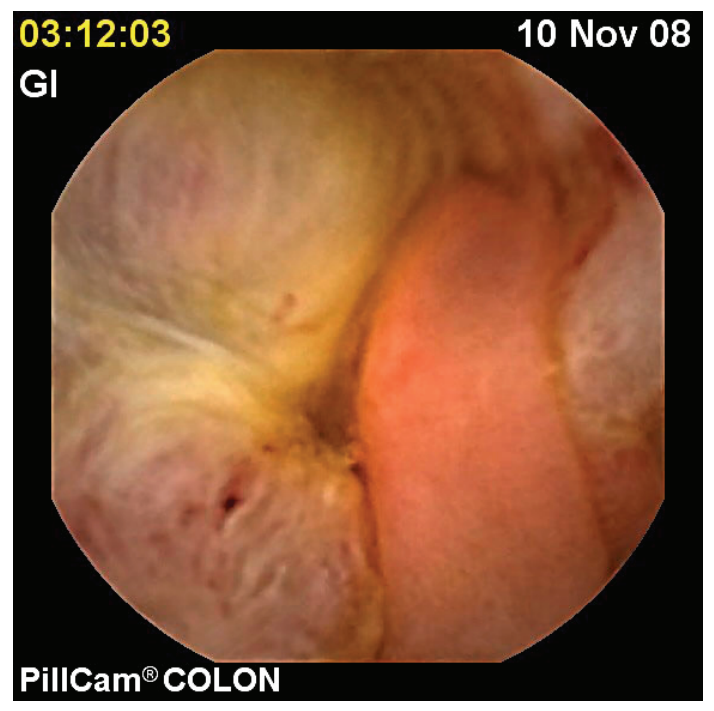


Fig. 2. Capsule endoscopic view revealing spontaneous tumor bleeding and stuck capsule position.

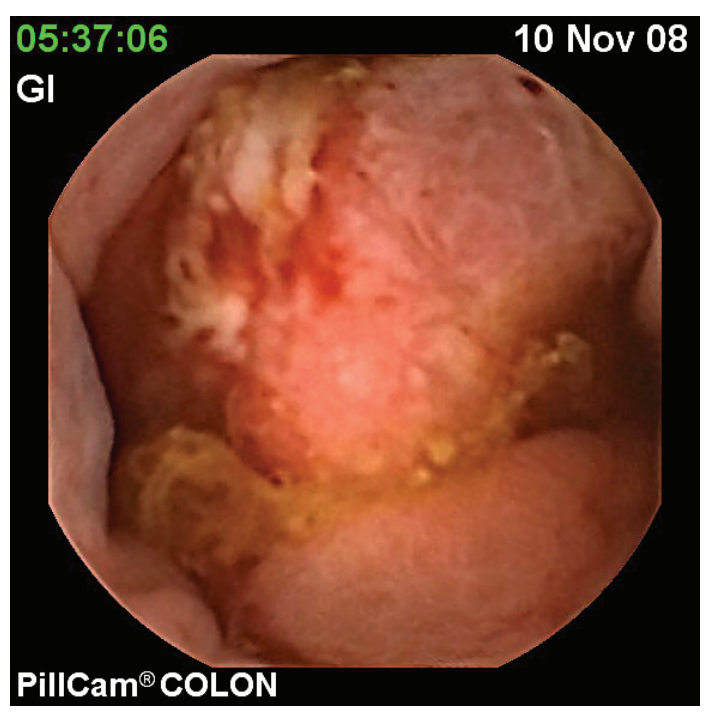

Fig. 3. a Colonoscopic view of the colon capsule stuck in the most narrow segment of the tumorous stricture. $\mathbf{b}$ The capsule was caught and passed through the stricture by a polypectomy snare.

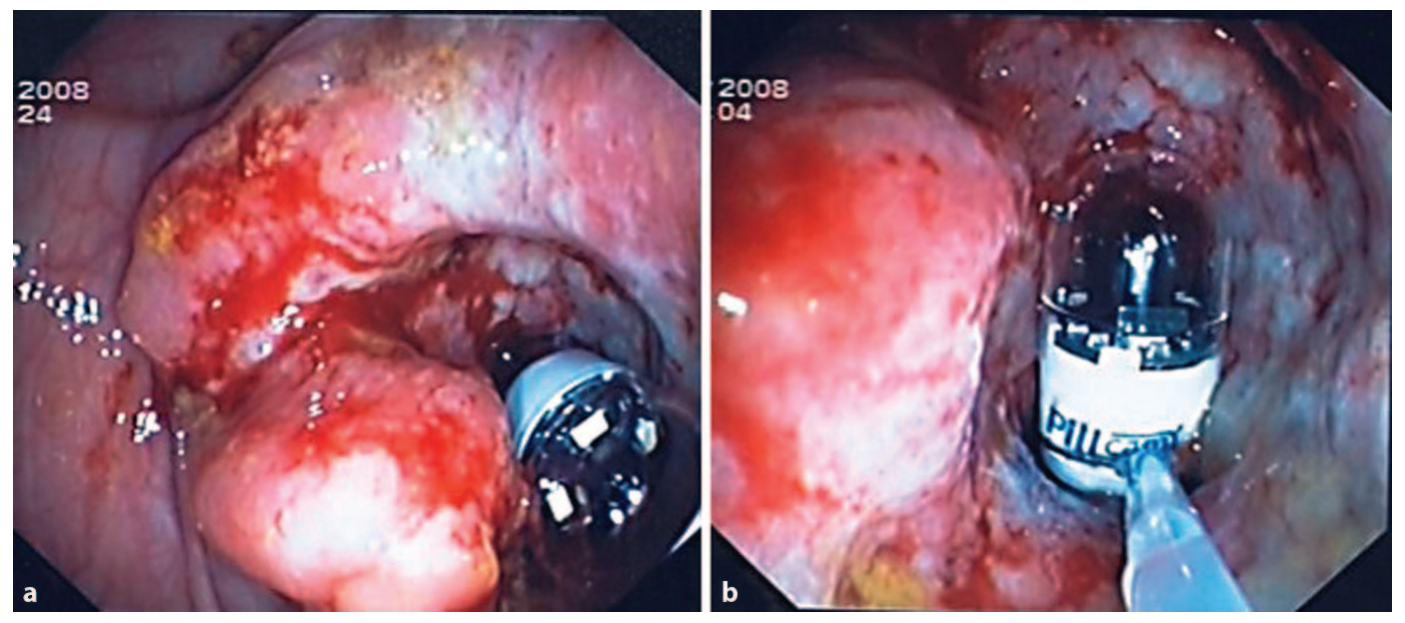


Fig. 4. a The capsule was temporary released in order to take biopsies. $\mathbf{b}$ The capsule was removed from the colon by re-catching with the polypectomy snare.

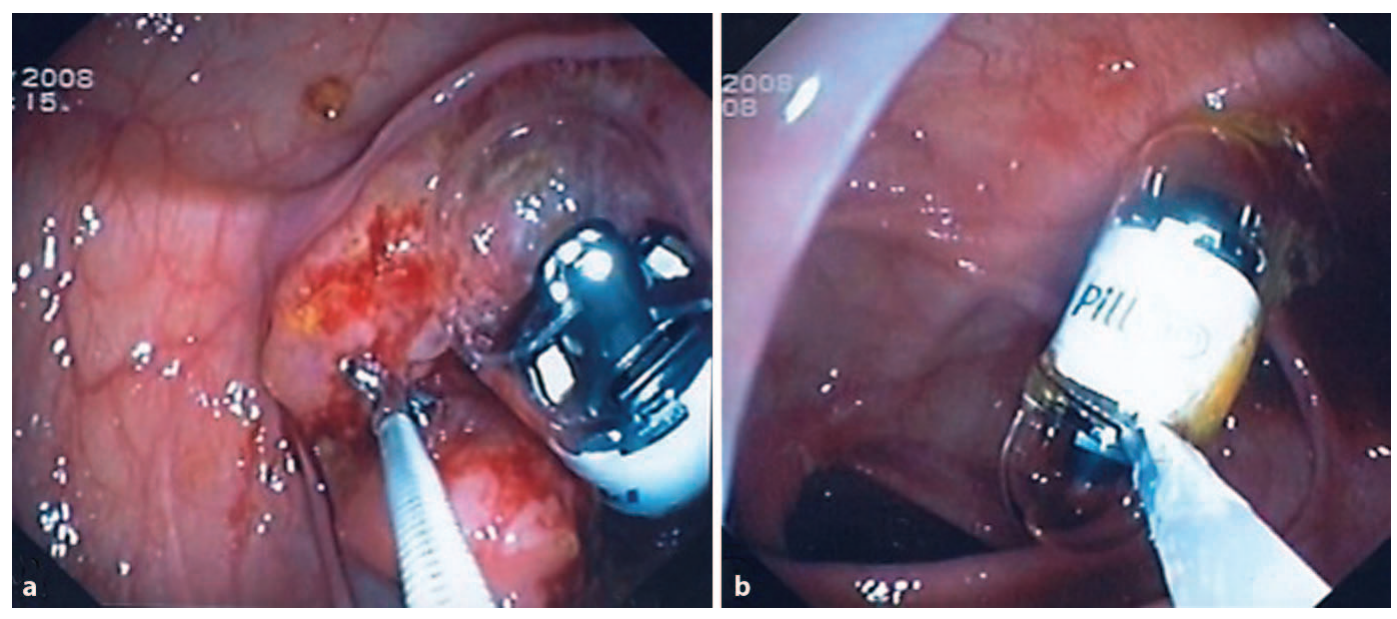




\section{References}

1 Eliakim R, Fireman Z, Gralnek IM, et al: Evaluation of the PillCam Colon capsule in the detection of colonic pathology: results of the first multicenter, prospective, comparative study. Endoscopy 2006;38:963-970.

-2 Schoofs N, Deviere J, van Gossum A: PillCam Colon capsule compared with colonoscopy for colorectal tumor diagnosis: a prospective pilot study. Endoscopy 2006;38:971-977.

-3 Deviere J, Munoz-Navas MA, Fernandez-Urien I, et al: PillCam Colon capsule endoscopy compared with colonoscopy in detection of colon polyps and cancers. Gastroenterology 2008;134:A-38.

- 4 Li F, Gurudu SR, De Petris G, et al: Retention of the capsule endoscope: a single-center experience of 1000 capsule endoscopy procedures. Gastrointest Endosc 2008;68:174-180.

5 Sears DM, Avots-Avotins A, Culp K, et al: Frequency and clinical outcomes of capsule retention during capsule endoscopy for GI bleeding of obscure origin. Gastrointest Endosc 2004;60:822-827.

-6 Cave D, Legnani P, de Franchis R, et al: ICCE consensus for capsule retention. Endoscopy 2005;37:1065-1067.

-7 Mishkin DS, Chuatti R, Croffie J, et al: ASGE technology status evaluation report: wireless capsule endoscopy. Gastrointest Endosc 2006;65:539-545.

-8 Riccioni ME, Shah S, Urgesi R, et al: Case report: A lesson in capsule endoscopy. Hepatogastroenterology 2008;55:1006-1007.

-9 Spada C, Riccioni ME, Petruziello L, et al: The new PillCam Colon capsule: difficult colonoscopy? No longer a problem? Gastrointest Endosc 2008;68:807808.

10 Triantafyllou K, Tsibouris P, Kalantzis C, et al: PillCam Colon capsule endoscopy does not always complement incomplete colonoscopy. Gastrointest Endosc 2009;69:572-576. 